\title{
THE GOERITZ MATRIX AND SIGNATURE OF A TWO BRIDGE KNOT
}

\author{
MICHAEL GALLASPY AND STANISLAV JABUKA
}

\begin{abstract}
According to a formula by Gordon and Litherland [5], the signature $\sigma(K)$ of a knot $K$ can be computed as $\sigma(K)=\sigma(G)-\mu$, where $G$ is the Goeritz matrix of a projection $D$ of $K$ and $\mu$ is a "correction term," read off from the projection $D$. In this article, we consider the family of two bridge knots $K_{p / q}$ and compute the signature of the Goeritz matrices of their "standard projections," $D_{p / q}$, by explicitly diagonalizing the Goertiz matrix over the rationals. We give applications to signature computations and concordance questions.
\end{abstract}

\section{Introduction.}

1.1. Definitions and results. Given a collection of nonzero integers $c_{1}, \ldots, c_{n} \in \mathbb{Z}$, the associated two bridge knot/link $K_{\left[c_{1}, c_{2}, \ldots, c_{n}\right]}$ is the isotopy class of the knot diagram $D_{\left[c_{1}, \ldots, c_{n}\right]}$ as in Figure 1. To the ordered collection $\left(c_{1}, \ldots, c_{n}\right)$ we associate a rational number $p / q$ by means of its continued fraction expansion:

$$
\left[c_{1}, \ldots, c_{n}\right]:=c_{1}-\frac{1}{c_{2}-\frac{1}{\ddots-\frac{1}{c_{n-1}-\frac{1}{c_{n}}}}}
$$

and write $p / q=\left[c_{1}, \ldots, c_{n}\right]$. It is a remarkable theorem of Conway [3] that, if two continued fractions $\left[c_{1}, \ldots, c_{n}\right]$ and $\left[d_{1}, \ldots, d_{m}\right]$ yield the same rational number $p / q$, then the two knots/links $K_{\left[c_{1}, \ldots, c_{n}\right]}$

2010 AMS Mathematics subject classification. Primary 57M25, 57M27.

The first author was supported by an REU supplement to the second author's NSF grant. The second author was partially supported by NSF grant DMS-0709625 and by grant no. 246123 from the Simons Foundation.

Received by the editors on August 2, 2013.

DOI:10.1216/RMJ-2015-45-4-1119 Copyright (C)2015 Rocky Mountain Mathematics Consortium 
and $K_{\left[d_{1}, \ldots, d_{m}\right]}$ are isotopic. This justifies the notation $K_{p / q}$ instead of $K_{\left[c_{1}, \ldots, c_{n}\right]}$, which we shall employ when convenient.

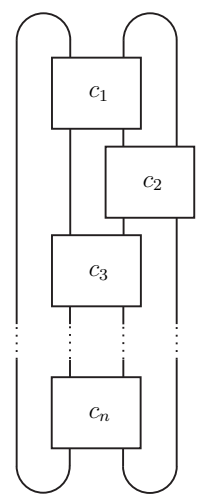

Case of $n$ odd.

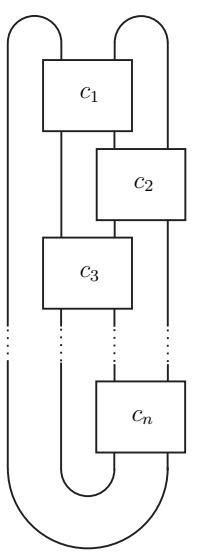

Case of $n$ even.

Figure 1. The two bridge knot/link associated to the nonzero integers $c_{1}, \ldots, c_{n} \in \mathbb{Z}$. The meaning of each box containing an integer is as in Figure 2. Throughout this article, we shall use the symbol $D_{\left[c_{1}, \ldots, c_{n}\right]}$ to denote this particular projection of the knot/link $K_{\left[c_{1}, \ldots, c_{n}\right]}$.

Before stating our main results, we pause to define the notion of a canonical representation of a continued fraction (see [7, subsection 1.2]). Given the equation $\left[c_{1}, \ldots, c_{n}\right]=p_{n} / q_{n}$, the choice of integers $p_{n}, q_{n}$ is of course not unique. However, we define a canonical choice of $p_{n}$ and $q_{n}$ for each $\left[c_{1}, \ldots, c_{n}\right]$, by induction on $n$, as follows. If $n=1$, then set $p_{1}=c_{1}$ and $q_{1}=1$. Suppose the canonical representations of all continued fractions of length $n-1$ have been defined. Then we declare $p_{n}$ and $q_{n}$, the canonical representation of $\left[c_{1}, \ldots, c_{n}\right]$, as given by

$$
p_{n}=c_{1} p_{n-1}^{\prime}-q_{n-1}^{\prime} \quad \text { and } \quad q_{n}=p_{n-1}^{\prime},
$$

where $p_{n-1}^{\prime}$ and $q_{n-1}^{\prime}$ are the canonical representatives of $\left[c_{2}, \ldots, c_{n}\right]$. 

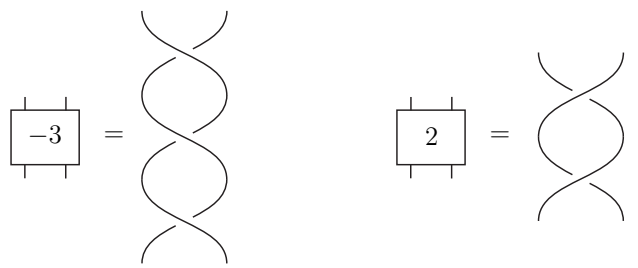

Figure 2. Each of the boxes from Figure 1, containing an integer $c$ and two incoming/outgoing strings, represents a pair of parallel strands with $|c|$ half-twists. Our convention is that $c>0$ corresponds to right-handed and $c<0$ to left-handed half-twists.

To make our definition plausible, note that

$$
\begin{aligned}
{\left[c_{1}, \ldots, c_{n}\right] } & =c_{1}-\frac{1}{\left[c_{2}, \ldots, c_{n}\right]}=c_{1}-\frac{1}{p_{n-1}^{\prime} / q_{n-1}^{\prime}} \\
& =\frac{c_{1} p_{n-1}^{\prime}-q_{n-1}^{\prime}}{p_{n-1}^{\prime}}=\frac{p_{n}}{q_{n}} .
\end{aligned}
$$

From here on out, whenever we write $\left[c_{1}, \ldots, c_{n}\right]=p / q$, we shall take $p$ and $q$ to be the canonical representatives of $\left[c_{1}, \ldots, c_{n}\right]$ without explicit mention.

Remark 1.1. Since $\left[c_{1}, \ldots, c_{n}\right]=\left[c_{1}, \ldots, c_{n} \pm 1, \pm 1\right]$, we can always assume, without loss of generality, that a knot $K_{p / q}$ equals $K_{\left[c_{1}, \ldots, c_{n}\right]}$ with $n$ odd.

The Goeritz form $G=G(D)$ associated to a particular projection $D$ of the knot $K$ is a symmetric, bilinear, non-degenerate form $G$ : $\mathbb{Z}^{N} \times \mathbb{Z}^{N} \rightarrow \mathbb{Z}$ where $N$ depends on the diagram $D$ (for the benefit of the reader, we recount the definition of the Goeritz form $G$ in Section 2). By means of choosing a basis for $\mathbb{Z}^{N}$, we will allow ourselves to view $G$ as an $N \times N$ symmetric non-degenerate matrix, referred to as the Goeritz matrix. As such, it can be diagonalized over the rationals, that is, there exists a matrix $P \in G l_{N}(\mathbb{Q})$ such that $P^{\tau} G P=\operatorname{Diag}\left(a_{1}, \ldots, a_{N}\right)$. We 
shall capture such a statement by writing

$$
P^{\tau} G P=\left\langle a_{1}\right\rangle \oplus\left\langle a_{2}\right\rangle \oplus \cdots \oplus\left\langle a_{N}\right\rangle,
$$

where $\langle a\rangle$ should be thought of as a matrix representative of a bilinear form on a one-dimensional rational vector space. With this in mind, our main result is contained in the next theorem.

Theorem 1.2. Let $K=K_{\left[c_{1}, \ldots, c_{n}\right]}$ be the two bridge knot/link associated to the collection $\left(c_{1}, \ldots, c_{n}\right)$ of ordered, nonzero integers, and assume that $n$ is odd (see Remark 1.1). Let $G$ be the Goeritz matrix of $K$ associated to its projection $D_{\left[c_{1}, \ldots, c_{n}\right]}$ as in Figure 1.

Then there is a matrix $P \in G l_{N}(\mathbb{Q})$, where $N=\left|c_{1}\right|+\left|c_{3}\right|+\cdots+$ $\left|c_{n}\right|-1$, with $\operatorname{det} P= \pm 1$, such that

$$
\begin{aligned}
P^{\tau} G P & =\bigoplus_{i=1,3,5, \ldots, n}\left(\oplus_{k=1}^{\left|c_{i}\right|-1}\left\langle-\operatorname{Sign}\left(c_{i}\right) \frac{k+1}{k}\right\rangle\right) \\
& \oplus \bigoplus_{i=2,4, \ldots, n-1}\left\langle\frac{p_{i+1}}{c_{i+1} p_{i-1}}\right\rangle .
\end{aligned}
$$

Here $p_{m}$ is the numerator of the canonical representation of $\left[c_{1}, \ldots, c_{m}\right]=$ $p_{m} / q_{m}, m \leq n$. Accordingly, the signature $\sigma(G)$ of the Goeritz matrix $G$ is given by

$$
\sigma(G)=\sum_{i=1,3, \ldots, n}\left(\operatorname{Sign}\left(c_{i}\right)-c_{i}\right)+\sum_{i=2,4, \ldots, n-1} \operatorname{Sign}\left(\frac{p_{i+1}}{c_{i+1} p_{i-1}}\right) .
$$

By the Gordon-Litherland formula [5], the signature $\sigma(K)$ of a knot $K$ can be computed as

$$
\sigma(K)=\sigma(G)-\mu,
$$

where $G=G(D)$ is the Goeritz matrix of $K$ associated to a diagram $D$, and $\mu=\mu(D)$ is a "correction term," also read off from $D$ (we provide a detailed description of $\mu$ in Section 2).

Definition 1.3. A continued fraction $\left[c_{1}, \ldots, c_{n}\right]$ shall be called an even continued fraction if $n$ is odd and if each $c_{2 i}$ is even for $i=$ $1, \ldots,(n-1) / 2$. 
Each two bridge knot $K_{p / q}$ can be represented by a diagram $D_{\left[c_{1}, \ldots, c_{n}\right]}$ with $\left[c_{1}, \ldots, c_{n}\right]$ an even continued fraction. This has already been noted by Burde and Zieschang in [1, Proposition 12.17], but we give an alternative algorithm in Section 4 . We recount the details of the Burde-Zieschang algorithm in Section 4 and draw comparisons to our algorithm in Examples 4.5 and 4.6. The benefit of working with even continued fractions $\left[c_{1}, \ldots, c_{n}\right]$ is that the associated correction term $\mu\left(D_{\left[c_{1}, \ldots, c_{n}\right]}\right)$ vanishes (Lemma 4.1), leading to the following effective signature computation for two bridge knots.

Corollary 1.4. Let $\left[c_{1}, \ldots, c_{n}\right]$ be an even continued fraction expansion of $p / q$. Then

$$
\sigma\left(K_{p / q}\right)=\sum_{i=1,3, \ldots, n}\left(\operatorname{Sign}\left(c_{i}\right)-c_{i}\right)+\sum_{i=2,4, \ldots, n-1} \operatorname{Sign}\left(\frac{p_{i+1}}{c_{i+1} p_{i-1}}\right) .
$$

In this formula, $p_{i} / q_{i}$ are the canonical representatives of $\left[c_{1}, \ldots, c_{i}\right]$, $i \leq n$.

Remark 1.5. Our orientation conventions are such that the righthanded trefoil $K_{3 / 1}$ has signature -2 , which is the opposite convention of those used in $[\mathbf{1}, \mathbf{1 1}]$.

Remark 1.6. The aforementioned Proposition 12.17 in [1] gives an algorithm for finding a unique even continued fraction expansion for $q / p$ (with $q$ odd and with $0<q<p$ ), of the form

$$
\frac{q}{p}=\frac{1}{c_{1}+\frac{1}{c_{2}+\frac{1}{\ddots+\frac{1}{c_{n}}}}}
$$

with $n$ odd, and with $c_{2}, c_{4}, \ldots, c_{n-1}$ all even (in our notation this would give the even continued fraction expansion $p / q=\left[c_{1},-c_{2}, c_{3},-c_{4}, c_{5}, \ldots\right.$, $\left.\left.-c_{n-1}, c_{n}\right]\right)$. We shall write $\left[c_{1}, \ldots, c_{n}\right]_{B Z}$ for the continued fraction expansion (1.3) to distinguish it from the one in (1.1) and refer to it 
as the Burde-Zieschang even continued fraction expansion, or BurdeZieschang expansion, for short. Burde and Zieschang in [1, Exercise E12.5 ] ask the reader to verify the signature formula (changed here to reflect our orientation convention, see Remark 1.5)

$$
\sigma\left(K_{p / q}\right)=\operatorname{Sign}\left(c_{n}\right)-\left(\sum_{i=1,3, \ldots, n} c_{i}\right) .
$$

While this formula is simpler than equation (1.2), it does not hold for general even continued fraction expansions. The difference

$$
\sum_{i=2,4, \ldots, n-1}\left[\operatorname{Sign}\left(c_{i-1}\right)+\operatorname{Sign}\left(\frac{p_{i+1}}{c_{i+1} p_{i-1}}\right)\right]
$$

between the right-hand sides of (1.2) and (1.4) is easily seen not to vanish for all even continued fraction expansions, compare to Example 4.6 (but for the Burde-Zieschang expansion, each term

$$
\operatorname{Sign}\left(c_{i-1}\right)+\operatorname{Sign}\left(\frac{p_{i+1}}{c_{i+1} p_{i-1}}\right), \quad i=1,3, \ldots, n-2
$$

of this sum vanishes separately).

Another advantage of using Corollary 1.4 over equation (1.4) for signature computations of two bridge knots becomes apparent when starting with an even continued fraction expansion associated to a collection of integer $c_{1}, \ldots, c_{n}$, rather than with a rational number $p / q$. There is no a priori way of telling whether the integers $c_{1}, \ldots, c_{n}$ constitute a Burde-Zieschang expansion, and so to use (1.4), one would first have to find the rational number $q / p$ associated to $\left[c_{1}, \ldots, c_{n}\right]_{B Z}$, compute its associated Burde-Zieschang expansion $\left[d_{1}, \ldots, d_{m}\right]_{B Z}$ and then use equation (1.4). In contrast, when using formula (1.2), these intermediate steps are not necessary, and one can compute the signature directly from $\left[c_{1}, \ldots, c_{n}\right]$.

In summary, our signature formula (1.2) generalizes that of Burde and Zieschang in that it applies to all even continued fraction expansions and reduces to (1.4) for the Burde Zieschang expansion.

1.2. Applications to signature computations. The primary utility of Theorem 1.2 and Corollary 1.4 is an effective and speedy algorithm for computing signatures of two-bridge knots. A formula for 
computing the signature of $K_{p / q}$ can be found in Murasugi's book [11], by which one

(i) forms the sequence $\{0, q, 2 q, 3 q, \ldots,(p-1) q\}$,

(ii) writes $j q=2 \ell_{j} p+r_{j}$ for $j=0, \ldots, p-1$, and with $\ell_{j} \in \mathbb{Z}$, $r_{j} \in\{-p+1, \ldots, p-1\}$

(iii) forms the new sequence $\left\{0, r_{1}, r_{2}, \ldots, r_{p-1}\right\}$,

(iv) and computes the signature $\sigma\left(K_{p / q}\right)$ as the number of positive entries minus the number of negative entries in the sequence from (iii).

Strictly speaking, Murasugi's algorithm assumes that $0<q<p$ and that $q$ is odd, both of which can always be achieved for any two bridge knot. A Mathematica program realizing Murasugi's signature computation can be downloaded from the second author's web page.

The formula for $\sigma\left(K_{\left[c_{1}, \ldots, c_{n}\right]}\right)$ provided by Theorem 1.2 and Corollary 1.4 is rather different in nature, relying on the coefficients of the continued fraction expansion of $p / q$ rather than $p$ and $q$ themselves. This can lead to significantly shorter computations in some examples. For instance, considering the knots $K_{3023 / 151}$ and $K_{52587 / 4825}$ and using the even continued fraction expansions

$$
3023 / 151=[20,-50,3] \text { and } 52587 / 4825=[11,10,9,8,7],
$$

Corollary 1.4 readily yields

$$
\begin{aligned}
\sigma\left(K_{3023 / 151}\right)= & {[(\operatorname{Sign}(20)-20)+(\operatorname{Sign}(3)-3)]+\operatorname{Sign}\left(\frac{p_{3}}{3 \cdot p_{1}}\right) } \\
= & -21+\operatorname{Sign}\left(\frac{-3023}{3 \cdot 20}\right) \\
= & -22 . \\
\sigma\left(K_{52587 / 4825}\right)= & {[(\operatorname{Sign}(11)-11)+(\operatorname{Sign}(9)-9)+(\operatorname{Sign}(7)-7)] } \\
& +\operatorname{Sign}\left(\frac{p_{3}}{9 \cdot p_{1}}\right)+\operatorname{Sign}\left(\frac{p_{5}}{7 \cdot p_{3}}\right) \\
= & -24+\operatorname{Sign}\left(\frac{970}{9 \cdot 11}\right)+\operatorname{Sign}\left(\frac{52587}{7 \cdot 970}\right) \\
= & -22 .
\end{aligned}
$$

In comparison, the algorithm from [11] described above requires forming lists of remainders with 3023 and 52587 entries, respectively, and 
counting the number of positive/negative elements. The advantage of our approach becomes more prominent the larger $|p|$ is.

We also point to the paper [4] by Curtis and Taylor for another signature formula, one that uses the Jones polynomial and boundary slopes as input.

Recall that an oriented knot is a knot with a chosen string orientation. On a projection of the knot this is indicated by decorating it with an arrow, Figure 3. Two oriented knots $K_{1}$ and $K_{2}$ can be joined into a connected sum, yielding a new knot denoted by $K_{1} \# K_{2}$. This is accomplished by removing an unknotted open $\operatorname{arc} I_{i}$ from $K_{i}$ and joining the boundary points of $K_{1}-I_{1}$ to those of $K_{2}-I_{2}$ in a way that respects the orientation on each knot, see Figure 4.

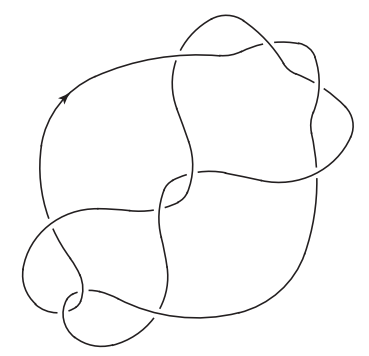

FIGURE 3. Example of an oriented knot, in this case of the knot $10_{13}$ from the knot tables [2].
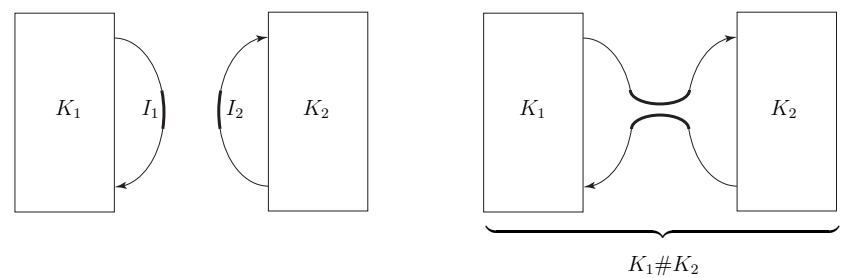

Figure 4 . The connected sum $K_{1} \# K_{2}$ of two oriented knots $K_{1}$ and $K_{2}$. 
An oriented knot $K$ is called smoothly slice (or slice for brevity) if it is the oriented boundary of a smoothly and properly embedded 2-disk in the 4-ball $D^{4}$. Two oriented knots $K_{1}$ and $K_{2}$ are called concordant if $-K_{1} \# K_{2}$ is slice (where $-K_{1}$ is the reverse mirror of $K_{1}$ ). The notion of concordance is an equivalence relation, and its equivalence classes, under the operation \# of connect summing, form an Abelian group $\mathcal{C}$ called the concordance group. The concordance group $\mathcal{C}$ is a central object in low-dimensional topology with relevance and applications to the theory of 3-manifolds and smooth 4-manifolds. Even so, it remains rather poorly understood (see [6] for a survey of recent results); not even the possible types of torsion elements of $\mathcal{C}$ are known.

While the subgroup of $\mathcal{C}$ generated by two bridge knots is not known, Lisca $[\mathbf{9}, \mathbf{1 0}]$ was able to obtain a complete list of slice two bridge knots as well as a complete list of slice knots among twofold sums $K_{1} \# K_{2}$ of two bridge knots. Beyond this, little is known about when a sum $K_{1} \# \cdots \# K_{n}$ of two bridge knots is slice. Since slice knots have signature zero and $\sigma\left(K_{1} \# \cdots \# K_{n}\right)=\sigma\left(K_{1}\right)+\cdots+\sigma\left(K_{n}\right)$, Theorem 1.2 and Corollary 1.4 provide a computable obstruction to the sliceness of $K_{1} \# K_{2} \# \cdots \# K_{n}$. Here are a few examples illustrating this principle.

Example 1.7. Consider the knots $K_{1}=K_{35 / 13}, K_{2}=K_{283 / 317}$ and $K_{3}=K_{1193 / 145}$. Then neither of the knots

$$
\left( \pm K_{1}\right) \#\left( \pm K_{2}\right) \#\left( \pm K_{3}\right)
$$

can be slice. Since $35 / 13=[3,4,2,2,2], 283 / 317=[1,10,2,2,12]$ and $1193 / 145=[9,2,2,2,4,2,-6]$, Corollary 1.4 shows that

$$
\sigma\left(K_{35 / 16}\right)=-2, \quad \sigma\left(K_{283 / 317}\right)=-10, \quad \sigma\left(K_{1193 / 145}\right)=-4,
$$

from which the claim follows.

Example 1.8. Let $K_{1}=K_{187 / 213}, K_{2}=K_{1451 / 131}$ and $K_{3}=K_{715 / 23}$. Then the knot $K_{1} \#\left(n \cdot K_{2}\right) \#\left(m \cdot K_{3}\right)$ cannot be slice for any choice of $m, n \in \mathbb{Z}$. Here $n \cdot K$ stands for the $n$-fold connected sum of $K$ with itself. 
Using Corollary 1.4, we obtain the signature of $K_{1}, K_{2}$ and $K_{3}$ :

$$
\begin{array}{ll}
\frac{187}{213}=[1,8,-6,-2,-2,-2,-2] & \Longrightarrow \sigma\left(K_{1}\right)=6, \\
\frac{1451}{131}=[11,-14,-2,-2,-2,-2,-2,-2,-2,-2,-2] & \Longrightarrow \sigma\left(K_{2}\right)=-10, \\
\frac{715}{23}=[31,-12,-2] & \Longrightarrow \sigma\left(K_{3}\right)=-30 .
\end{array}
$$

Thus, the signature of $K_{1} \#\left(n \cdot K_{2}\right) \#\left(m \cdot K_{3}\right)$ is $6-10(n+3 m)$ which is nonzero for any choice of $n, m \in \mathbb{Z}$.

1.3. Organization. The remainder of this article is organized as follows. Section 2 reviews the definitions of the Goeritz matrix $G$ and the correction term $\mu$ associated to a knot diagram $D$. Section 3 is devoted to the proof of Theorem 1.2 while the final Section 4 addresses Corollary 1.4. Section 4 also provides an explicit algorithm for finding an even continued fraction expansion for any given two bridge knot.

2. The Goeritz matrix $G$ and the correction term $\mu$. This section elucidates the definitions of the Goeritz matrix $G=G(D)$ and the correction term $\mu=\mu(D)$, both associated to a projection $D$ of a knot $K$. Our exposition follows the introductory section from [5].

Let $K$ be an oriented knot, and let $D$ be a projection of $K$. We color the regions of $D$ black and white, giving it a checkerboard pattern. Our convention is that the unbounded region of $D$ receive a white coloring, see Figure 5 for an example.

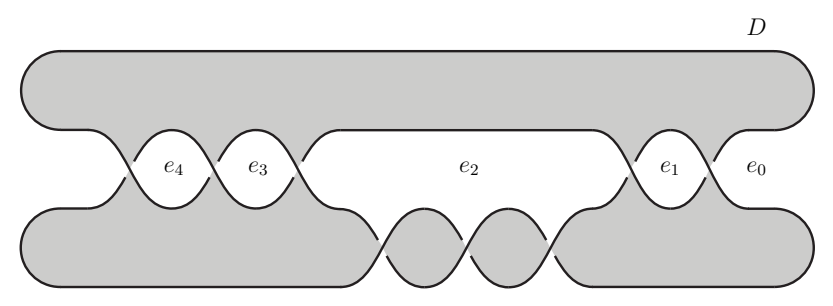

FIGURE 5. The checkerboard black-and-white coloring of the regions of this diagram $D$ of the knot $K=8_{6}$ from the knot tables [2]. Observe that $D=D_{[2,-3,3]}$, showing that $K$ is the two bridge knot $K_{23 / 10}$. 
To each crossing $p_{i}$ in the diagram $D$, we associate two pieces of data, the sign of the crossing $\eta\left(p_{i}\right)$ and the type of the crossing $\tau\left(p_{i}\right)$, both of which are defined in Figure 6 .

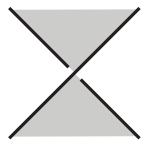

$\eta(p)=1$

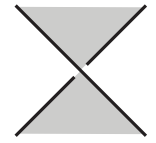

$\eta(p)=-1$

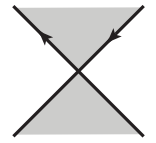

$\tau(p)=\mathrm{I}$

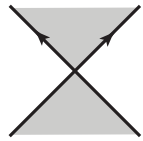

$\tau(p)=\mathrm{II}$

Figure 6 . The functions $\eta$ and $\tau$ assign to a double point $p$ the values \pm 1 and types I/II, respectively. Note that $\eta(p)$ only depends on over/undercrossing information about $p$ while $\tau(p)$ only depends on the orientation of the diagram $D$ near $p$.

Let $\left\{e_{0}, \ldots, e_{N}\right\}$ be a labeling of the white regions in the checkerboard pattern of $D$, with the convention that $e_{0}$ labels the unbounded region, and let $\mathbb{Z}^{N+1}$ be the free Abelian group generated by these symbols. Then the pre-Goeritz form $P G$ is a bilinear symmetric form $P G: \mathbb{Z}^{N+1} \times \mathbb{Z}^{N+1} \rightarrow \mathbb{Z}$ whose associated matrix $\left[g_{i j}\right]$ with respect to the ordered basis $\left\{e_{0}, \ldots, e_{N}\right\}$, is given by

$$
g_{i j}= \begin{cases}-\sum_{p \in e_{i} \cap e_{j}} \eta(p) ; & i \neq j \\ -\sum_{k \neq i} g_{i k} ; & i=j .\end{cases}
$$

The sum $\sum_{p \in e_{i} \cap e_{j}} \eta(p)$ is over all double points $p$ that connect the two white regions $e_{i}$ and $e_{j}$ in the diagram $D$. The Goeritz form $G$ is obtained by restricting the form $P G$ to $\mathbb{Z}^{N} \times \mathbb{Z}^{N}$ where $\mathbb{Z}^{N} \subset \mathbb{Z}^{N+1}$ is obtained by discarding the $\mathbb{Z}$ summand generated by $e_{0}$. Since this construction relies on the choice of a basis of $\mathbb{Z}^{N}$, namely, $\left\{e_{1}, \ldots, e_{N}\right\}$, we can, and often shall, think of $G$ as a symmetric $N \times N$ square matrix (with integer entries), called the Goeritz matrix. It follows from the work in [5] that $|\operatorname{det} G|=\operatorname{det} K$ so that $G$ is in fact a regular matrix. 
For simplicity of notation, we adopt the following convention which will substantially simplify our computations in the next section:

$$
G(a, b)=\langle a, b\rangle \quad \text { for all } a, b \in \mathbb{Z}^{N} .
$$

For example, the Goeritz matrix associated to the diagram $D$ and the basis $\left\{e_{1}, e_{2}, e_{3}, e_{4}\right\}$ from Figure 5 is given by

$$
G=\left[\begin{array}{rrrr}
-2 & 1 & 0 & 0 \\
1 & -5 & 1 & 0 \\
0 & 1 & -2 & 1 \\
0 & 0 & 1 & -2
\end{array}\right]
$$

We leave it as an exercise to show that the signature of this matrix is $\sigma(G)=-4$, and its determinant is $\operatorname{det} G=23$.

The correction term $\mu=\mu(D)$ associated to an oriented knot diagram $D$ is computed as

$$
\mu=\sum_{\tau(p)=\mathrm{II}} \eta(p) .
$$

In the above, the sum is taken over all double points $p$ of $D$ that are of type II. For example, for the diagram $D$ from Figure 5 , one finds $\mu=-2$ (the only type II crossings are those adjacent to the region $e_{1}$ ).

With these understood, the following is proved in [5].

Theorem 2.1 (Gordon-Litherland [5]). Given any oriented diagram $D$ of a knot $K$, the signature $\sigma(K)$ of $K$ can be computed as

$$
\sigma(K)=\sigma(G)-\mu \text {. }
$$

Here $G$ and $\mu$ are the Goeritz matrix and the correction term associated to $D$.

Returning to the example from Figure 5, we compute the signature of the knot $K=8_{6}$ by means of the above theorem:

$$
\sigma\left(8_{6}\right)=\sigma(G)-\mu=-4-(-2)=-2 .
$$

Alternatively, since the knot $8_{6}$ is the two bridge knot $K_{23 / 10}\left(=K_{23 / 33}\right)$ (see Figure 5) and since $23 / 33=[1,4,2,2,4]$, Corollary 1.4 also shows that $\sigma\left(K_{23 / 10}\right)=-2$. 
3. The proof of Theorem 1.2. This section is devoted to the proof of Theorem 1.2. We first compute the Goeritz matrix associated to the specific diagram $D=D_{\left[c_{1}, \ldots, c_{n}\right]}$ utilized in Figure 1 and then proceed to diagonalize it by employing the Gram-Schmidt process.

Let $n>0$ be an odd integer (compare Remark 1.1), let $c_{1}, \ldots, c_{n}$ be a collection of nonzero integers, and let $K=K_{\left[c_{1}, \ldots, c_{n}\right]}$ be the associated two bridge knot. Let $D=D_{\left[c_{1}, \ldots, c_{n}\right]}$ be the diagram of $K$ as in Figure 7 (see also Figure 1).

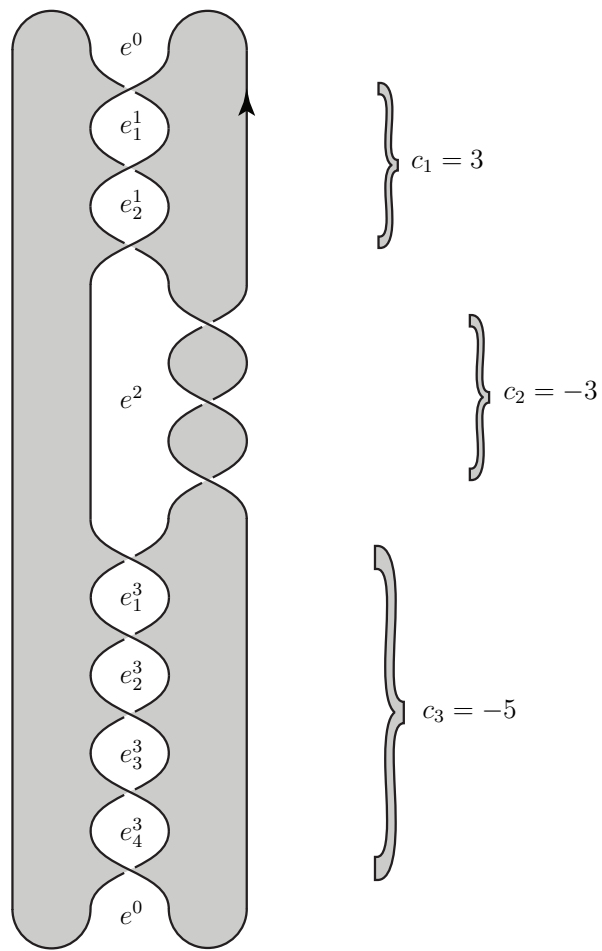

FIGURE 7 . The two bridge knot $K_{47 / 14}$ where $47 / 14=[3,-3,-5]$.

Give $D$ a checkerboard coloring and label its white regions as 
$e^{0}, e^{2}, \ldots, e^{n-1}$ and $e_{1}^{1}, \ldots, e_{\left|c_{1}\right|-1}^{1}, \ldots, e_{1}^{n}, \ldots, e_{\left|c_{n}\right|-1}^{n}$, where the labels are chosen as:

$$
\begin{aligned}
e^{0} & =\text { unbounded white region, } \\
e^{2 i} & =\text { white region adjacent to the } c_{2 i} \text { half-twists } \\
e_{1}^{2 i+1}, \ldots, e_{\left|c_{2 i+1}\right|-1}^{2 i+1} & =\text { white regions adjacent to the } c_{2 i+1} \text { half-twists. }
\end{aligned}
$$

These account for all white regions of $D$ showing that there is exactly

$$
N+1=\left|c_{1}\right|+\left|c_{3}\right|+\cdots+\left|c_{n}\right|
$$

of them (we express this number as $N+1$ since the region $e^{0}$ is discarded eventually when passing from the pre-Goertiz to the Goeritz matrix). The ordering of this basis for $\mathbb{Z}^{N}$ that we prefer to use is

$$
\left\{e^{0}, e_{1}^{1}, \ldots, e_{\left|c_{1}\right|-1}^{1}, e_{1}^{3}, \ldots, e_{\left|c_{3}\right|-1}^{3}, \ldots, e_{1}^{n}, \ldots, e_{\left|c_{n}\right|-1}^{n}, e^{2}, e^{4}, \ldots, e^{n-1}\right\}
$$

Recall our convention (2.1) by which we write $\langle a, b\rangle$ for $G(a, b)$. With this in mind, it is easy to see, by consulting Figure 7 , that (with $\left.\epsilon_{i}=\operatorname{Sign}\left(c_{i}\right)\right)$

$$
\begin{aligned}
& \left\langle e_{k}^{i}, e_{\ell}^{j}\right\rangle=\left\{\begin{array}{cl}
-2 \epsilon_{i} ; & i=j \text { and }|k-\ell|=0 \\
\epsilon_{i} ; & i=j \text { and }|k-\ell|=1, \\
0 ; & i \neq j \text { or }|k-\ell| \geq 2,
\end{array}\right. \\
& \left\langle e^{i}, e_{\ell}^{j}\right\rangle=\left\{\begin{array}{cc}
-\epsilon_{i-1} ; & j=i-1 \text { and } \ell=\left|c_{i-1}\right|-1, \\
-\epsilon_{i+1} ; & j=i+1 \text { and } \ell=1, \\
0 ; & \text { otherwise, }
\end{array}\right. \\
& \left\langle e^{i}, e^{j}\right\rangle=\left\{\begin{array}{cc}
c_{i}-\epsilon_{i-1}-\epsilon_{i+1} ; & i=j \\
0 ; & i \neq j .
\end{array}\right.
\end{aligned}
$$

The above relations capture the Goeritz matrix $G$ associated to the diagram $D=D_{\left[c_{1}, \ldots, c_{n}\right]}$ : 


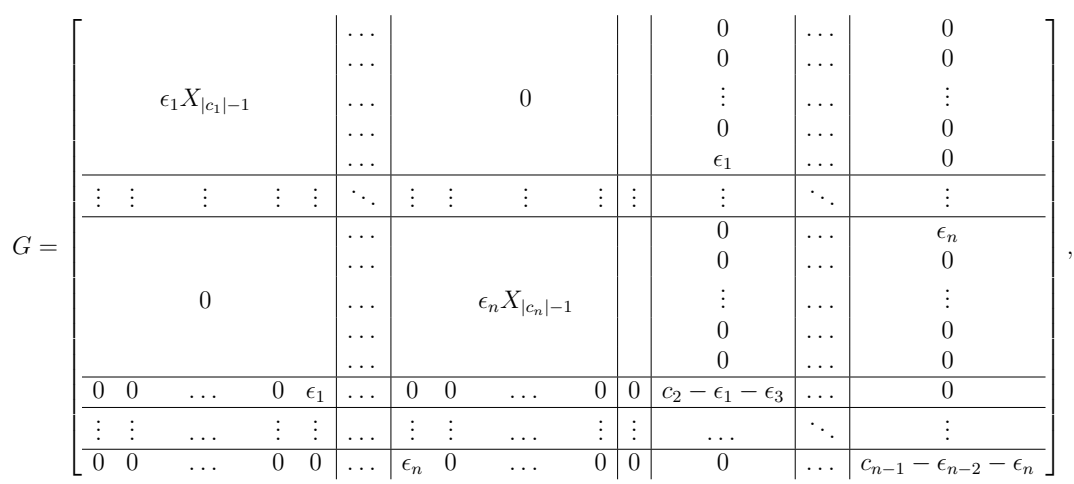

FiguRe 8.

The symbol $X_{m}$, utilized in the description of $G$, denotes the $m \times m$ square matrix

$$
X_{m}=\left[\begin{array}{rrrrrrr}
-2 & 1 & 0 & 0 & \cdots & 0 & 0 \\
1 & -2 & 1 & 0 & \cdots & 0 & 0 \\
0 & 1 & -2 & 1 & \cdots & 0 & 0 \\
0 & 0 & 1 & -2 & \cdots & 0 & 0 \\
\vdots & \vdots & \vdots & \vdots & \ddots & \vdots & \vdots \\
0 & 0 & 0 & 0 & \cdots & 1 & 0 \\
0 & 0 & 0 & 0 & \cdots & -2 & 1 \\
0 & 0 & 0 & 0 & \cdots & 1 & -2
\end{array}\right]
$$

We now turn to the task of diagonalizing $G$. We do so by thinking of $G: \mathbb{Z}^{N} \times \mathbb{Z}^{N} \rightarrow \mathbb{Z}$ as a bilinear form, one whose matrix description (Figure 8 ) is a facet of having chosen the basis

$$
\begin{array}{r}
\mathcal{E}=\left\{e_{1}^{1}, \ldots, e_{\left|c_{1}\right|-1}^{1}, e_{1}^{3}, \ldots, e_{\left|c_{3}\right|-1}^{3}, \ldots, e_{1}^{n}, \ldots, e_{\left|c_{n}\right|-1}^{n},\right. \\
\left.e^{2}, e^{4}, \ldots, e^{n-1}\right\}
\end{array}
$$

for $\mathbb{Z}^{N}$. Our task then becomes to find a new basis for $\mathbb{Z}^{N}$, one with respect to which $G$ has a diagonal matrix representative. The new basis 


$$
\begin{array}{r}
\mathcal{F}=\left\{f_{1}^{1}, \ldots, f_{\left|c_{1}\right|-1}^{1}, f_{1}^{3}, \ldots, f_{\left|c_{3}\right|-1}^{3}, \ldots, f_{1}^{n}, \ldots, f_{\left|c_{n}\right|-1}^{n},\right. \\
\left.f^{2}, f^{4}, \ldots, f^{n-1}\right\}
\end{array}
$$

will be obtained in several steps, outlined in Lemmas 3.1-3.4, each of which follows the Gram-Schmidt procedure. By way of nomenclature, we shall say that $a, b \in \mathbb{Z}^{N}$ are orthogonal if $\langle a, b\rangle=0$ (that is if $G(a, b)=0)$. A subset $A \subset \mathbb{Z}^{N}$ is orthogonal if $\langle a, b\rangle=0$ for all $a, b \in A$.

Lemma 3.1. For any choice of $i \in\{1,3,5, \ldots, n\}$ and $k \in\left\{1, \ldots,\left|c_{i}\right|-\right.$ $1\}$, let $f_{k}^{i}$ be defined as

$$
f_{k}^{i}=\frac{\epsilon_{i}}{k}\left(e_{1}^{i}+2 e_{2}^{i}+3 e_{3}^{i}+\cdots+k e_{k}^{i}\right) .
$$

Then the set $\left\{f_{k}^{i}\right\}_{k=1, \ldots,\left|c_{i}\right|-1}^{i=1,3, \ldots, n}$ is orthogonal and

$$
\left\langle f_{k}^{i}, f_{k}^{i}\right\rangle=-\epsilon_{i} \frac{k+1}{k} .
$$

Proof. Since $\left\langle e_{k}^{i}, e_{\ell}^{j}\right\rangle=0$ whenever $i \neq j$, it follows that $\left\langle f_{k}^{i}, f_{\ell}^{j}\right\rangle=0$ for all $i \neq j$. When $i=j$, pick two indices $k<\ell$ from $\left\{1, \ldots,\left|c_{i}\right|-1\right\}$. Then

$$
\begin{aligned}
k \ell \cdot\left\langle f_{k}^{i}, f_{\ell}^{i}\right\rangle & =\left\langle\sum_{r=1}^{k} r e_{r}^{i}, \sum_{s=1}^{\ell} s e_{s}^{i}\right\rangle \\
& =\left\langle e_{1}^{i}, e_{1}^{i}+2 e_{2}^{i}\right\rangle+\sum_{r=2}^{k}\left(\left\langle r e_{r}^{i},(r-1) e_{r-1}^{i}+r e_{r}^{i}+(r+1) e_{r+1}^{i}\right\rangle\right. \\
& =\epsilon_{i} \sum_{r=2}^{k} r(r-1)-2 r^{2}+r(r+1) \\
& =0 .
\end{aligned}
$$

Similarly, computing $\left\langle f_{k}^{i}, f_{k}^{i}\right\rangle$ gives

$$
\begin{aligned}
k^{2} \cdot\left\langle f_{k}^{i}, f_{k}^{i}\right\rangle & =\left\langle\sum_{r=1}^{k} r e_{r}^{i}, \sum_{s=1}^{k} s e_{s}^{i}\right\rangle \\
& =\left\langle e_{1}^{i}, e_{1}^{i}+2 e_{2}^{i}\right\rangle+\left\langle k e_{k}^{i},(k-1) e_{k-1}^{i}+k e_{k}^{i}\right\rangle
\end{aligned}
$$




$$
\begin{aligned}
& +\sum_{r=2}^{k-1}\left(\left\langle r e_{r}^{i},(r-1) e_{r-1}^{i}+r e_{r}^{i}+(r+1) e_{r+1}^{i}\right\rangle\right. \\
= & \epsilon_{i}\left(k(k-1)-2 k^{2}\right) \\
= & -\epsilon_{i} k(k+1),
\end{aligned}
$$

as claimed.

Before proceeding, we remark that the relations (3.1) and the definition of $f_{k}^{i}$ (3.4) imply the following

$$
\left\langle f_{k}^{i}, e^{j}\right\rangle= \begin{cases}1 ; & j=i+1 \text { and } k=\left|c_{i}\right|-1, \\ \frac{1}{k} ; & j=i-1 \text { and } k=1, \ldots,\left|c_{i}\right|-1, \\ 0 ; & \text { otherwise. }\end{cases}
$$

We define the remaining elements $f^{2}, f^{4}, \ldots, f^{n-1} \in \mathbb{Z}^{N}$ for the basis $\mathcal{F}$ from (3.3) in two steps. The next lemma first defines elements $\widehat{f}^{2}, \ldots, \widehat{f}^{n-1}$, each of which is orthogonal to the previously defined $f_{k}^{i}$ and with $\left\langle\widehat{f}^{i}, \widehat{f}^{j}\right\rangle=0$ whenever $|i-j| \geq 4$. These $\widehat{f}^{i}$ shall then be further modified in Lemma 3.3 to obtain the desired $f^{i}$.

Lemma 3.2. For $j=2,4,6, \ldots, n-1$, we define $\widehat{f}^{j}$ as

$$
\widehat{f}^{j}=e^{j}+\frac{\left|c_{j-1}\right|-1}{c_{j-1}} f_{\left|c_{j-1}\right|-1}^{j-1}+\sum_{k=1}^{\left|c_{j+1}\right|-1} \frac{\epsilon_{j+1}}{k+1} f_{k}^{j+1} .
$$

Then, each $\widehat{f}^{j}$ is orthogonal to the set $\left\{f_{k}^{i}\right\}_{k=1, \ldots,\left|c_{i}\right|-1}^{i=1,3, \ldots, n}$, and additionally

$$
\left\langle\widehat{f}^{i}, \widehat{f}^{j}\right\rangle=\left\{\begin{array}{cl}
c_{j}-\frac{1}{c_{j-1}}-\frac{1}{c_{j+1}} ; & j=i, \\
\frac{1}{c_{i \pm 1}} ; & j=i \pm 2, \\
0 ; & \text { otherwise. }
\end{array}\right.
$$

Proof. All of these are direct computations, some of which make implicit use of the formulas from (3.5). To begin with, note that $\left\langle\widehat{f}^{j}, f_{k}^{i}\right\rangle=0$ whenever $i \neq j \pm 1$. For $i=j-1$, we similarly have that $\left\langle\widehat{f}^{j}, f_{k}^{j-1}\right\rangle=0$ if $k \neq\left|c_{j-1}\right|-1$, while if $k=\left|c_{j-1}\right|-1$ then

$$
\left\langle\widehat{f}^{j}, f_{\left|c_{j-1}\right|-1}^{j-1}\right\rangle=\left\langle e^{j}+\frac{1}{c_{j-1}} f_{\left|c_{j-1}\right|-1}^{j-1}+\sum_{k=1}^{\left|c_{j+1}\right|-1} \frac{\epsilon_{j+1}}{k+1} f_{k}^{j+1}, f_{\left|c_{j-1}\right|-1}^{j-1}\right\rangle
$$




$$
\begin{aligned}
& =\left\langle e^{j}, f_{\left|c_{j-1}\right|-1}^{j-1}\right\rangle+\frac{1}{c_{j-1}}\left\langle f_{\left|c_{j-1}\right|-1}^{j-1}, f_{\left|c_{j-1}\right|-1}^{j-1}\right\rangle \\
& =1+\left(\frac{\left|c_{j-1}\right|-1}{c_{j-1}} \cdot\left(-\epsilon_{j-1}\right) \frac{\left|c_{j-1}\right|}{\left|c_{j-1}\right|-1}\right) \\
& =0 .
\end{aligned}
$$

Turning to the same computation with $i=j+1$, we find

$$
\begin{aligned}
\left\langle\widehat{f}^{j}, f_{\ell}^{j+1}\right\rangle & =\left\langle e^{j}+\frac{\left|c_{j-1}\right|-1}{c_{j-1}} f_{\left|c_{j-1}\right|-1}^{j-1}+\sum_{k=1}^{\left|c_{j+1}\right|-1} \frac{\epsilon_{j+1}}{k+1} f_{k}^{j+1}, f_{\ell}^{j+1}\right\rangle \\
& =\left\langle e^{j}, f_{\ell}^{j+1}\right\rangle+\sum_{k=1}^{\left|c_{j+1}\right|-1} \frac{\epsilon_{j+1}}{k+1}\left\langle f_{k}^{j+1}, f_{\ell}^{j+1}\right\rangle \\
& =\frac{1}{\ell}+\frac{\epsilon_{j+1}}{\ell+1}\left\langle f_{\ell}^{j+1}, f_{\ell}^{j+1}\right\rangle \\
& =\frac{1}{\ell}+\frac{\epsilon_{j+1}}{\ell+1} \cdot\left(-\epsilon_{j+1}\right) \frac{\ell+1}{\ell} \\
& =0 .
\end{aligned}
$$

These last two calculations verify that $\widehat{f}^{j}$ is orthogonal to $f_{k}^{i}$ for any choice of $i, k$. From the definition of $\widehat{f}^{j}$, it follows that $\left\langle\widehat{f}^{j}, \widehat{f}^{i}\right\rangle=0$ whenever $|j-i|>2$. When $i=j-2$, the following computation proves one of the remaining claims of the lemma:

$$
\begin{aligned}
\left\langle\widehat{f}^{j}, \widehat{f}^{j-2}\right\rangle & \\
= & \left\langle e^{j}+\frac{\left|c_{j-1}\right|-1}{c_{j-1}} f_{\left|c_{j-1}\right|-1}^{j-1}+\sum_{k=1}^{\left|c_{j+1}\right|-1} \frac{\epsilon_{j+1}}{k+1} f_{k}^{j+1},\right. \\
\left.e^{j-2}+\frac{\left|c_{j-3}\right|-1}{c_{j-3}} f_{\left|c_{j-3}\right|-1}^{j-3}+\sum_{k=1}^{\left|c_{j-1}\right|-1} \frac{\epsilon_{j-1}}{k+1} f_{k}^{j-1}\right\rangle & \\
(3.6)= & \left\langle e^{j}, \frac{\epsilon_{j-1}}{\left|c_{j-1}\right|} f_{\left|c_{j-1}\right|-1}^{j-1}\right\rangle+\left\langle\frac{\left|c_{j-1}\right|-1}{c_{j-1}} f_{\left|c_{j-1}\right|-1}^{j-1}, e^{j-2}\right\rangle \\
& +\left\langle\frac{\left|c_{j-1}\right|-1}{c_{j-1}} f_{\left|c_{j-1}\right|-1}^{j-1}, \frac{\epsilon_{j-1}}{\left|c_{j-1}\right|} f_{\left|c_{j-1}\right|-1}^{j-1}\right\rangle \\
= & \frac{1}{c_{j-1}}+\frac{1}{c_{j-1}}-\frac{1}{c_{j-1}} \\
= & \frac{1}{c_{j-1}} .
\end{aligned}
$$


The very last computation is that of $\left\langle\widehat{f}^{i}, \widehat{f}^{i}\right\rangle$, to which we now turn.

$$
\begin{aligned}
& \left\langle\widehat{f}^{i}, \widehat{f}^{i}\right\rangle=\left\langle e^{i}, e^{i}\right\rangle+\frac{\left(\left|c_{i-1}\right|-1\right)^{2}}{c_{i-1}^{2}}\left\langle f_{\left|c_{i-1}\right|-1}^{i-1}, f_{\left|c_{i-1}\right|-1}^{i-1}\right\rangle \\
& +\sum_{k=1}^{\left|c_{i+1}\right|-1} \frac{1}{(k+1)^{2}}\left\langle f_{k}^{i+1}, f_{k}^{i+1}\right\rangle \\
& +2 \frac{\left|c_{i-1}\right|-1}{c_{i-1}}\left\langle e^{i}, f_{\left|c_{i-1}\right|-1}^{i-1}\right\rangle+2 \sum_{k=1}^{\left|c_{i+1}\right|-1} \frac{\epsilon_{i+1}}{k+1}\left\langle e^{i}, f_{k}^{i+1}\right\rangle \\
& =\left\langle e^{i}, e^{i}\right\rangle-\frac{\left|c_{i-1}\right|-1}{c_{i-1}}-\epsilon_{i+1} \sum_{k=1}^{\left|c_{i+1}\right|-1} \frac{1}{k(k+1)} \\
& +2 \frac{\left|c_{i-1}\right|-1}{c_{i-1}}+\epsilon_{i+1} \sum_{k=1}^{\left|c_{i+1}\right|-1} \frac{2}{k(k+1)} \\
& =\left\langle e^{i}, e^{i}\right\rangle+\epsilon_{i-1} \frac{\left|c_{i-1}\right|-1}{\left|c_{i-1}\right|}+\epsilon_{i+1} \sum_{k=1}^{\left|c_{i+1}\right|-1} \frac{1}{k(k+1)} \\
& =\left(c_{i}-\epsilon_{i-1}-\epsilon_{i+1}\right)+\epsilon_{i-1}\left(1-\frac{1}{\left|c_{i-1}\right|}\right) \\
& +\epsilon_{i+1}\left(1-\frac{1}{\left|c_{i+1}\right|}\right) \\
& =c^{i}-\frac{1}{c_{i-1}}-\frac{1}{c_{i+1}} \text {. }
\end{aligned}
$$

This completes the proof of the lemma.

Lemma 3.3. Define the sequence $\lambda_{2 j} \in \mathbb{Q}, j \geq 1$ recursively as

$$
\lambda_{2}=c_{2}-\frac{1}{c_{1}}-\frac{1}{c_{3}}
$$

and

$$
\lambda_{2 j}=\left(c_{2 j}-\frac{1}{c_{2 j-1}}-\frac{1}{c_{2 j+1}}\right)-\frac{1}{c_{2 j-1}^{2} \cdot \lambda_{2 j-2}}, \quad j \geq 2
$$


Using this sequence, we define the vectors $f^{j}$, for $j=2,4, \ldots, n-1$, as

$$
f^{2}=\widehat{f}^{2} \quad \text { and } \quad f^{j}=\widehat{f}^{j}-\frac{1}{c_{j-1} \cdot \lambda_{j-2}} f^{j-2} \quad \text { for } j \geq 4 .
$$

Then the set $\left\{f_{k}^{i}, f^{j}\right\}_{k=1,2, \ldots,\left|c_{i}\right|-1}^{i=1,3, \ldots, n ; j=2,4, \ldots, n-1}$ is orthogonal and $\left\langle f^{j}, f^{j}\right\rangle=$ $\lambda_{j}$.

Proof. The proof of this lemma is a tedious computation. It should be clear that $f^{j}$ is orthogonal to $f_{k}^{i}$ for any choices of $i, j, k$. To verify the claim about $\left\langle f^{j}, f^{i}\right\rangle$, we proceed by induction on $j$ (and assume that $i \leq j)$.

Since $f^{2}=\widehat{f}^{2}$, it follows from Lemma 3.2 that $\left\langle f^{2}, f^{2}\right\rangle=\lambda_{2}$. Taking $j=4$, we obtain

$$
\begin{aligned}
\left\langle f^{4}, f^{2}\right\rangle & =\left\langle\widehat{f}^{4}-\frac{1}{c_{3} \cdot \lambda_{2}} f^{2}, \widehat{f}^{2}\right\rangle \\
& =\left\langle\widehat{f}^{4}, \widehat{f}^{2}\right\rangle-\frac{1}{c_{3} \cdot \lambda_{2}}\left\langle\widehat{f}^{2}, \widehat{f}^{2}\right\rangle \\
& =\frac{1}{c_{3}}-\frac{1}{c_{3} \cdot \lambda_{2}} \lambda_{2} \\
& =0 . \\
\left\langle f^{4}, f^{4}\right\rangle & =\left\langle\widehat{f}^{4}-\frac{1}{c_{3} \cdot \lambda_{2}} \widehat{f}^{2}, \widehat{f}^{4}-\frac{1}{c_{3} \cdot \lambda_{2}} \widehat{f}^{2}\right\rangle \\
& =\left\langle\widehat{f}^{4}, \widehat{f}^{4}\right\rangle-\frac{2}{c_{3} \cdot \lambda_{2}}\left\langle\widehat{f}^{4}, \widehat{f}^{2}\right\rangle+\frac{1}{\left(c_{3} \cdot \lambda_{2}\right)^{2}}\left\langle\widehat{f}^{2}, \widehat{f}^{2}\right\rangle \\
& =\left(c_{4}-\frac{1}{c_{3}}-\frac{1}{c_{5}}\right)-\frac{2}{\left(c_{3}\right)^{2} \cdot \lambda_{2}}+\frac{1}{\left(c_{3} \cdot \lambda_{2}\right)^{2}} \lambda_{2} \\
& =\left(c_{4}-\frac{1}{c_{3}}-\frac{1}{c_{5}}\right)-\frac{1}{\left(c_{3}\right)^{2} \cdot \lambda_{2}} \\
& =\lambda_{4} .
\end{aligned}
$$

For the step of induction, we suppose the lemma to be true for all $2 i, 2 j \leq 2 m-2$ and turn to computing $\left\langle f^{2 m}, f^{2 i}\right\rangle$ (with $i \leq m$ ). Firstly, suppose that $2 i \leq 2 m-4$ :

$$
\left\langle f^{2 m}, f^{2 i}\right\rangle=\left\langle\hat{f}^{2 m}-\frac{1}{c_{2 m-1} \cdot \lambda_{2 m-2}} f^{2 m-2}, f^{2 i}\right\rangle=0 .
$$


Next, let's take $2 i=2 m-2$ :

$$
\begin{aligned}
\left\langle f^{2 m}, f^{2 m-2}\right\rangle & =\left\langle\widehat{f}^{2 m}-\frac{1}{c_{2 m-1} \cdot \lambda_{2 m-2}} f^{2 m-2}, f^{2 m-2}\right\rangle \\
& =\left\langle\widehat{f}^{2 m}, f^{2 m-2}\right\rangle-\frac{1}{c_{2 m-1} \cdot \lambda_{2 m-2}}\left\langle f^{2 m-2}, f^{2 m-2}\right\rangle \\
& =\left\langle\widehat{f}^{2 m}, \widehat{f}^{2 m-2}-\frac{1}{c_{2 m-3} \cdot \lambda_{2 m-4}} \widehat{f}^{2 m-4}\right\rangle-\frac{1}{c_{2 m-1}} \\
& =\frac{1}{c_{2 m-1}}-\frac{1}{c_{2 m-1}} \\
& =0 .
\end{aligned}
$$

It remains to address the case of $2 i=2 m$ :

$$
\begin{aligned}
\left\langle f^{2 m}, f^{2 m}\right\rangle= & \left\langle\widehat{f}^{2 m}-\frac{1}{c_{2 m-1} \cdot \lambda_{2 m-2}} f^{2 m-2}, \widehat{f}^{2 m}-\frac{1}{c_{2 m-1} \cdot \lambda_{2 m-2}} f^{2 m-2}\right\rangle \\
(3.7) & \left\langle\widehat{f}^{2 m}, \widehat{f}^{2 m}\right\rangle-\frac{2}{c_{2 m-1} \cdot \lambda_{2 m-2}}\left\langle f^{2 m-2}, \widehat{f}^{2 m}\right\rangle \\
& +\frac{1}{\left(c_{2 m-1} \cdot \lambda_{2 m-2}\right)^{2}}\left\langle f^{2 m-2}, f^{2 m-2}\right\rangle \\
= & \left(c_{2 m}-\frac{1}{c_{2 m-1}}-\frac{1}{c_{2 m+1}}\right)-\frac{2}{\left(c_{2 m-1}\right)^{2} \cdot \lambda_{2 m-2}} \\
& +\frac{\lambda_{2 m-2}}{\left(c_{2 m-1} \cdot \lambda_{2 m-2}\right)^{2}} \\
= & \left(c_{2 m}-\frac{1}{c_{2 m-1}}-\frac{1}{c_{2 m+1}}\right)-\frac{1}{\left(c_{2 m-1}\right)^{2} \cdot \lambda_{2 m-2}} \\
= & \lambda_{2 m} .
\end{aligned}
$$

With this, the lemma is proved.

For the next lemma, the reader is asked to recall the definition of the canonical representation of the continued fraction $\left[c_{1}, \ldots, c_{n}\right]$ by the rational number $p_{n} / q_{n}$ (discussed in the introduction of Section 1).

Lemma 3.4. Let $c_{1}, c_{2}, \ldots, c_{n}$ (with $n$ odd) and $\lambda_{i}, i=2,4, \ldots, n-1$ be as in Lemma 3.3. For $i=1,2, \ldots, n$, let us introduce the relatively prime integers $p_{i}, q_{i}$ as the numerator and denominator of the canonical 
representation of $\left[c_{1}, \ldots, c_{i}\right]$ :

$$
\left[c_{1}, c_{2}, \ldots, c_{i}\right]=\frac{p_{i}}{q_{i}}
$$

Then

$$
\lambda_{2 i}=\frac{p_{2 i+1}}{c_{2 i+1} \cdot p_{2 i-1}} \quad \text { for each } i \in\left\{1,2, \ldots, \frac{n-1}{2}\right\}
$$

Proof. We start by noting the following recursive relations connecting the various $p_{i}$ and $q_{i}$ (see [7, Theorem 1]):

$$
p_{n}=c_{n} p_{n-1}-p_{n-2} \quad \text { and } \quad q_{n}=c_{n} q_{n-1}-q_{n-2} \text {. }
$$

Let us set $\mu_{2 i}=p_{2 i+1} /\left(c_{2 i+1} \cdot p_{2 i-1}\right)$. To show that $\lambda_{2 i}=\mu_{2 i}$, it suffices to demonstrate that $\mu_{2 i}$ satisfies the recursion relation

$$
\mu_{2}=c_{2}-\frac{1}{c_{1}}-\frac{1}{c_{3}}
$$

and

$$
\mu_{2 i}=\left(c_{2 i}-\frac{1}{c_{2 i-1}}-\frac{1}{c_{2 i+1}}\right)-\frac{1}{c_{2 i-1}^{2} \cdot \mu_{2 i-2}}
$$

from Lemma 3.3. The first of these equations is evident ( since $p_{1}=c_{1}$ and $\left.p_{3}=c_{1} c_{2} c_{3}-c_{1}-c_{3}\right)$. The second is established using the noted recursive relation for $p_{i}$ :

$$
\begin{aligned}
\mu_{2 i} & =\frac{p_{2 i+1}}{c_{2 i+1} p_{2 i-1}}=\frac{c_{2 i+1} p_{2 i}-p_{2 i-1}}{c_{2 i+1} p_{2 i-1}}=\frac{p_{2 i}}{p_{2 i-1}}-\frac{1}{c_{2 i+1}} \\
& =\frac{c_{2 i} p_{2 i-1}-p_{2 i-2}}{p_{2 i-1}}-\frac{1}{c_{2 i+1}} \\
& =c_{2 i}-\frac{1}{c_{2 i+1}}-\frac{p_{2 i-2}}{p_{2 i-1}} \\
& =c_{2 i}-\frac{1}{c_{2 i+1}}-\frac{c_{2 i-1} p_{2 i-2}}{c_{2 i-1} p_{2 i-1}} \\
& =c_{2 i}-\frac{1}{c_{2 i+1}}-\frac{p_{2 i-1}+p_{2 i-3}}{c_{2 i-1} p_{2 i-1}} \\
& =c_{2 i}-\frac{1}{c_{2 i+1}}-\frac{1}{c_{2 i-1}}-\frac{1}{c_{2 i-1}^{2} \cdot \frac{p_{2 i-1}}{c_{2 i-1} p_{2 i-3}}}
\end{aligned}
$$




$$
=c_{2 i}-\frac{1}{c_{2 i+1}}-\frac{1}{c_{2 i-1}}-\frac{1}{c_{2 i-1}^{2} \cdot \mu_{2 i-2}} .
$$

This completes the proof of the lemma.

Lemmas 3.1-3.4 provide a proof of Theorem 1.2. Namely, since the basis

$\mathcal{F}=\left\{f_{1}^{1}, \ldots, f_{\left|c_{1}\right|-1}^{1}, f_{1}^{3}, \ldots, f_{\left|c_{3}\right|-1}^{3}, \ldots, f_{1}^{n}, \ldots, f_{\left|c_{n}\right|-1}^{n}, f^{2}, f^{4}, \ldots, f^{n-1}\right\}$

is orthogonal with respect to the "inner product" $\langle\cdot, \cdot\rangle$ provided by $G$ (according to Lemma 3.3), it follows that the matrix representing $G$ with respect to the basis $\mathcal{F}$ is diagonal and its entries are $\left\{\left\langle f_{1}^{1}, f_{1}^{1}\right\rangle, \ldots,\left\langle f^{n-1}, f^{n-1}\right\rangle\right\}$. These latter quantities have been computed (Lemma 3.1 and Lemmas 3.3, 3.4) and are

$$
\left\langle f_{k}^{i}, f_{k}^{i}\right\rangle=-\epsilon_{i} \frac{k+1}{k} \text { and }\left\langle f^{2 j}, f^{2 j}\right\rangle=\frac{p_{2 i+1}}{c_{2 i+1} p_{2 i-1}} .
$$

This proves Theorem 1.2 after observing that the transition matrix $P$ from the old basis $\mathcal{E}(3.2)$ to the new basis $\mathcal{F}(3.3)$ of $\mathbb{Z}^{N}$, is upper triangular with \pm 1 entries on the diagonal.

\section{Proof of Corollary 1.4 and existence of even continued fractions.}

Lemma 4.1. Let $D_{\left[c_{1}, \ldots, c_{n}\right]}$ be the standard projection associated to an even continued fraction expansion $\left[c_{1}, \ldots, c_{n}\right]$. Then $\mu\left(D_{\left[c_{1}, \ldots, c_{n}\right]}\right)=0$.

The result of this lemma has can be deduced from [1, Remark 12.27] and the fact that the correction term vanishes for orientable Seifert surfaces. As the proof of the lemma is rather elementary we include it here for completeness and for the benefit of the reader.

Proof. Consider the surface bounding $D_{\left[c_{1}, \ldots, c_{n}\right]}$ from Figure 9 . It is easy to see that if $\left[c_{1}, \ldots, c_{n}\right]$ is an even continued fraction expansion, then the said surface is orientable and becomes a Seifert surface for $D_{\left[c_{1}, \ldots, c_{n}\right]}$. The lemma now follows in conduction with the observation from [5] that the correction term associated to an orientable surface vanishes. 


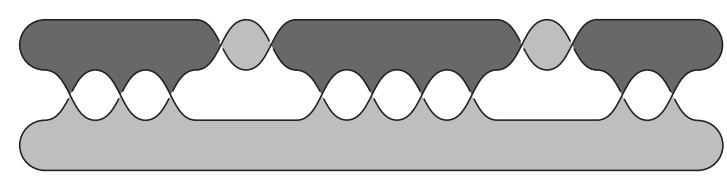

FIGURE 9. This is the diagram $D_{[3,-2,-4,-2,-2]}$ of the two bridge knot $K_{61 / 17}$ associated to the even continued fraction expansion $61 / 17=$ $[3,-2,-4,-2,-2]$. The oriented Seifert surface is formed by the shaded regions. The darker shaded regions represent one side of the surface, the lighter shaded regions the other.

Corollary 1.4 is now a direct consequence of Lemma 4.1 and Theorem 1.2.

As the usefulness of Corollary 1.4 rests on the existence of even continued fractions $\left[c_{1}, \ldots, c_{n}\right]$, we devote the rest of this section to describing two algorithms for finding such expansions. The first of these is a recount of the algorithm described by Burde and Zieschang in [1, Proposition 12.17].

Algorithm 4.2 (The Burde-Zieschang algorithm). Proposition 12.17 in [1] describes an algorithm for finding an even continued fraction expansions for a rational number $q / p=\left[c_{1}, \ldots, c_{n}\right]_{B Z}$ with $0<q<p$ and with $q$ odd (recall the symbol $\left[c_{1}, \ldots, c_{n}\right]_{B Z}$ from Remark 1.6). The integers $c_{1}, \ldots, c_{n}$, of which $c_{2}, c_{4}, \ldots, c_{n-1}$ are even, are the results of a generalized Euclidean algorithm, uniquely determined by the requirements:

(i) Set $r_{0}=p$ and $r_{1}=q$, and consider the equation

$$
r_{i-1}=c_{i} r_{i}+r_{i+1}
$$

for $i=1, \ldots, n$.

(ii) Use equation (4.1), with $r_{i-1}, r_{i}$ known, to determine $c_{i}$ and $r_{i+1}$ subject to the conditions:

- $r_{2 i}>0$ for all $i=1, \ldots,(n-1) / 2$.

- $c_{2 i}$ is even for all $i=1, \ldots,(n-1) / 2$.

- $\left|r_{i}\right|<\left|r_{i-1}\right|$ for all $i=1, \ldots, n-1$ and $\left|r_{n}\right| \leq\left|r_{n-1}\right|$.

- If $\left|r_{n}\right|=\left|r_{n-1}\right|$ then $c_{n} c_{n-1}>0$. 
Effectively, using equation (4.1) with $r_{i-1}$ and $r_{i}$ known, one chooses $r_{i+1}$ as follows, depending on the parity of $i$ :

- If $i$ is odd, pick $r_{i+1}$ as the modulus of $r_{i-1}$ with respect to $r_{i}$ (that is, $r_{i+1} \in\{0, \ldots, q-1\}$ and $r_{i-1}-r_{i+1}$ is divisible by $r_{i}$ ), and set $c_{i}=\left(r_{i-1}-r_{i+1}\right) / r_{i}$.

- If $i$ is even, consider the two possibilities for $r_{i+1}$, namely, the modulus of $r_{i-1}$ with respect to $r_{i}$, and that same modulus minus $r_{i}$. For each possibility for $r_{i+1}$, compute the corresponding $c_{i}$ as $c_{i}=\left(r_{i-1}-r_{i+1}\right) / r_{i}$. Of these two possibilities, pick the one that yields an even $c_{i}$.

The algorithm ends when $r_{i}$ becomes zero for an even coefficient $i$.

A Mathematica implementation of this algorithm can be found on the second author's web page.

The second algorithm below is due to the authors. After describing it, we look at examples comparing the two methods.

Algorithm 4.3. Let $p, q \in \mathbb{Z}$ be two relatively prime, non-zero integers. Then the algorithm outlined below shows that either

$$
\frac{p}{q} \quad \text { or } \quad \frac{p}{p+q}
$$

possesses an even continued fraction expansion. For convenience, and without loss of generality, assume that $q>0$. Our algorithm then proceeds in four steps.

Step 1. If $p / q$ is an integer, let $c_{1}=p / q$ and note that $p / q=\left[c_{1}\right]$ is an even continued fraction expansion of $p / q$, at which point the algorithm terminates.

Step 2. If $p / q$ is not an integer, let $\varepsilon_{-1}=\operatorname{Sign}(p)$, and replace $p$ by $|p|$ so that $p / q>0$. For uniformity of notation, we introduce the abbreviations $r_{-1}=p$ and $r_{0}=q$. Find integers $c_{1}, r_{1}$ from the equation $\varepsilon_{-1} r_{-1}=c_{1} r_{0} \pm r_{1}$, subject to the condition $0 \leq r_{1}<r_{0}$. The sign is chosen so as to make $c_{1}$ odd, and we set $\varepsilon_{0}=\mp 1$ to keep track 
of our sign choice. Notice that we obtain

$\frac{p}{q}=\frac{\varepsilon_{-1} r_{-1}}{r_{0}}=\frac{c_{1} r_{0} \pm r_{1}}{r_{0}}=c_{1} \pm \frac{r_{1}}{r_{0}}=c_{1}-\frac{1}{\mp r_{0} / r_{1}}=c_{1}-\frac{1}{\epsilon_{0} \cdot\left(r_{0} / r_{1}\right)}$.

Step 3. This step inductively repeats Step 2 until the remainder $r_{n}$ becomes zero. The one significant difference between Step 2 and Step 3 is that $c_{1}$ was chosen odd in Step 2, while we will choose $c_{n}, n \geq 2$, to be even in Step 3. Specifically, in the nth step, having previously found $c_{1}, \ldots, c_{n-1}, \varepsilon_{-1}, \ldots, \varepsilon_{n-2}$ and $r_{-1}, \ldots, r_{n-1}$, the equation

$$
\varepsilon_{n-2} r_{n-2}=c_{n} r_{n-1} \pm r_{n}
$$

uniquely determines integers $r_{n}$ and $c_{n}$ subject to $c_{n}$ being even and $0 \leq r_{n}<r_{n-1}$ (this can be arranged as along as $r_{n} \neq 0$ ). Set $\varepsilon_{n-1}=\mp 1$, that is set it to the opposite sign of the one appearing in equation (4.3).

By induction on $n$, it is easy to see that the formula

$$
\frac{p}{q}=c_{1}-\frac{1}{c_{2}-\frac{1}{c_{n-1}-\frac{1}{c_{n}-\frac{1}{\epsilon_{n-1} \cdot\left(\frac{r_{n-2}}{r_{n-1}}\right)}}}}
$$

holds whenever $r_{n} \neq 0$. This follows from (4.2) which serves as the basis of induction, and from equation (4.3) applied to the induction hypothesis:

$$
\frac{p}{q}=c_{1}-\frac{1}{c_{2}-\frac{1}{c_{n-1}-\frac{1}{\epsilon_{n-2} \cdot\left(\frac{r_{n-2}}{r_{n-1}}\right)}}}
$$

If $r_{n}=0$, then the parity of $c_{n}$ may not be chosen at will, but rather 
is determined by equation (4.3). In that case, relation (4.5) (with the help of (4.3)) leads to

$$
\frac{p}{q}=c_{1}-\frac{1}{c_{2}-\frac{1}{\ddots-\frac{1}{c_{n-1}-\frac{1}{c_{n}}}}}=\left[c_{1}, \ldots, c_{n}\right] .
$$

Since the sequence $r_{0}, r_{1}, r_{2}, \ldots$ is a strictly decreasing sequence of non-negative integers, this process yields $r_{n}=0$ after finitely many iterations, at which point we have produced a continued fraction expansion of $p / q$ as in (4.6). We point out that $c_{1}$ is odd and $c_{i}$ even for $i \geq 2$ with the possible exception of $c_{n}$. Note also that $c_{i} \neq 0$ for all $i$ since $r_{i-2}<r_{i-1}$ for $i \geq 2$ and since $c_{1}$ was chosen to be odd.

Step 4. We consider the continued fraction expansion $\left[c_{1}, \ldots, c_{n}\right]$ from Step 3. If $n$ is odd, this continued fraction expansion is even and the algorithm terminates. If $n$ is even and $c_{n}$ is odd, we change to the continued fraction expansion $\left[c_{1}, \ldots, c_{n} \pm 1, \pm 1\right]$ of $p / q$ which is an even continued fraction expansion, and the algorithm terminates. Finally, if $n$ is even and $c_{n}$ is even, then the continued fraction expansion

$$
\left[1,1+c_{1}, c_{2}, \ldots, c_{n}\right]
$$

is even, and its value is

$$
\left[1,1+c_{1}, \ldots, c_{n}\right]=1-\frac{1}{1+c_{1}-\frac{1}{\ddots-\frac{1}{c_{n-1}-\frac{1}{c_{n}}}}}
$$




$$
\begin{aligned}
& =1-\frac{1}{1+\left(c_{1}-\frac{1}{\ddots-\frac{1}{c_{n-1}-\frac{1}{c_{n}}}}\right)} \\
& =1-\frac{1}{1+\frac{p}{q}} \\
& =\frac{p}{p+q},
\end{aligned}
$$

completing the algorithm.

A Mathematica implementation of Algorithm 4.3 can also be obtained on the second author's web page.

Corollary 4.4. Each two bridge knot $K_{p / q}$ can be represented by a diagram $D_{\left[c_{1}, \ldots, c_{n}\right]}$ where $\left[c_{1}, \ldots, c_{n}\right]$ is an even continued fraction.

Proof. If $0<q<p$ and $q$ is odd, the Burde-Zieschang algorithm 4.2 may be used to obtain the Burde-Zieschang expansion $q / p=\left[c_{1}, \ldots, c_{n}\right]_{B Z}$. Then the continued fraction expansion $\left[c_{1},-c_{2}, c_{3},-c_{4}, c_{5}, \ldots,-c_{n-1}, c_{n}\right]$ is an even continued fraction expansion for $p / q$. Alternatively, Algorithm 4.3 yields an even continued fraction expansion for either $p / q$ or $p /(p+q)$, proving the corollary once again after observing that the knots $K_{p / q}$ and $K_{p /(p+q)}$ are isotopic, see [11].

Numerical experimentation shows that many times Algorithms 4.2 and 4.3 produce the same even continued fraction expansion, or at least expansions of equal length. There are, however, exceptions when one algorithm performs better than the other, in the sense of yielding a shorter continued fraction expansion. 
Example 4.5. Consider the relatively prime integers $p=122,227$ and $q=11,113$. Algorithms 4.2 and 4.3 applied to $p$ and $q$ give

$$
\begin{aligned}
& \frac{11,113}{122,227}=[3,2,-1,-2,1,2,-4]_{B Z}, \\
& \frac{122,227}{11,113}=[3,-2,-2,-4,-4] .
\end{aligned}
$$

Formula (1.4) for the Burde-Zieschang expansion, and formula (1.2) for the second expansion, each yield signature -10 for $K_{122227 / 11113}$.

Example 4.6. For $p=137$ and $q=37$, Algorithms 4.2 and 4.3 yield

$$
\begin{aligned}
& \frac{37}{137}=[10,2,-1,-692,-1,-2,1,2,2]_{B Z}, \\
& \frac{137}{37}=[11,694,-2,-4,2],
\end{aligned}
$$

each giving the value 0 for the signature of $K_{137 / 37}$. We note that formula (1.4), when applied to the second (non Burde-Zieschang expansion) yields the incorrect result for the signature of $K_{137 / 37}$ of 2 (compare with Remark 1.6).

Acknowledgments. We would like to thank the anonymous referee for many useful comments that have helped improved the quality of exposition. We thank her/him in particular for drawing our attention to parallels of our work to that presented in Burde-Zieschang [1].

\section{REFERENCES}

1. G. Burde and H. Zieschang, Knots, Second edition, de Gruyter Stud. Math. 5, Walter de Gruyter \& Co., Berlin, 2003.

2. J.C. Cha and C. Livingston, KnotInfo: Table of knot invariants, http://www.indiana.edu/ knotinfo (September 25, 2009).

3. J. Conway, An enumeration of knots and links, and some of their algebraic properties, 1970 Computational Problems in Abstract Algebra (Proc. Conf., Oxford, 1967) Pergamon, Oxford, 329-358.

4. C. Curtis and S. Taylor, The Jones polynomial and boundary slopes of alternating knots, J. Knot Theor. Ramifica. 20 (2011), 1345-1354.

5. C. Gordon and R.A. Litherland, On the signature of a link, Invent. Math. 47 (1978), 53-69.

6. S. Jabuka, Heegaard Floer homology and knot concordance: A survey of recent results, Glas. Math. 42 (2007), 237-256. 
7. A.Ya. Khinchin, Continued fractions, Reprint, Dover Publications, Inc., Mineola, NY, 1997.

8. Robion Kirby and Paul Melvin, Dedekind sums, $\mu$-invariants and the signature cocycle, Math. Ann. 299 (1994), 231-267.

9. P. Lisca, Lens spaces, rational balls and the ribbon conjecture, Geom. Topol. 11 (2007), 429-472.

10. Sums of lens spaces bounding rational balls, Alg. Geom. Topol. 7 (2007), 2141-2164.

11. K. Murasugi, Knot theory and its applications, Birkhauser Boston, Inc., Boston, MA, 1996.

Department of Mathematics and Statistics, University of Nevada, Reno NV 89557

Email address: gallasp2@unr.nevada.edu

Department of Mathematics and Statistics, University of Nevada, Reno NV 89557

Email address: jabuka@unr.edu 